\title{
Free flap surgery in osteoradionecrosis of the head and neck
}

\author{
Arshad Kaleem ${ }^{1}$, Paul Amailuk ${ }^{2}$, Paolo Cariati ${ }^{3}$, Ramzey Tursun ${ }^{1}$ \\ ${ }^{1}$ Division of Oral and Maxilofacial Surgery, Department of Surgery, University of Miami, Miami, FL, USA; ${ }^{2}$ Department of Oral \& Maxillofacial \\ Surgery, Gold Coast University Hospital, Southport, Queensland, Australia; ${ }^{3}$ Department of Oral \& Maxillofacial Surgery, Hospital General \\ Universitario de Albacete, Albacete, Spain \\ Contributions: (I) Conception and design: R Tursun, A Kaleem; (II) Administrative support: R Tursun; (III) Provision of study materials or patients: \\ R Tursun, A Kaleem; (IV) Collection and assembly of data: P Amaliuk, P Cariati; (V) Data analysis and interpretation: All authors; (VI) Manuscript \\ writing: All authors; (VII) Final approval of manuscript: All authors. \\ Correspondence to: Paolo Cariati, MD, PhD. Department of Oral \& Maxillofacial Surgery, Hospital General Universitario de Albacete, Albacete, \\ Spain. Email: paolocariati1@gmail.com; francocariati@hotmail.com.
}

\begin{abstract}
Osteoradionecrosis (ORN) following radiation therapy is a much-feared complication of the treatment of head and neck cancer. Free flap reconstruction in the ORN patient has specific challenges for the surgeon. From 2014 to 2020 the Head and Neck Microvascular unit of the Oral and Maxillofacial Surgery Division at Jackson Health/University of Miami treated 149 ORN patients with free flap reconstruction. The fibula free flap $(n=65)$ and anterior lateral thigh free flap $(6 n=1)$ were the workhorse flaps in our series comprising $44 \%$ and $41 \%$ of the total flap surgeries. A total of 13 cases (8.7\%) involved performing 2 flaps in the same operation. Thirty cases had recorded complications with one flap loss $(0.7 \%)$ and one case of partial necrosis $(0.7 \%)$ of the flap. The most common complications encountered were flap congestion with 8 cases $(5 \%), 5$ cases of flap dehiscence, 4 cases of neck hematoma $(2.6 \%)$ and 3 cases of infection of the flap reconstruction site (3\%). The average length of stay was 8.2 days. In this paper we present our approach to reconstruction in these patients and the lessons we have learned. Though this surgery is complex and has greater risks than free flap surgery in the "virgin" neck, our experience demonstrates that good outcomes can be achieved consistently with a structured approach.
\end{abstract}

Keywords: Osteoradionecrosis (ORN); microvascular reconstruction; free flaps; irradiated neck

Received: 01 March 2021; Accepted: 25 March 2021; Published: 10 June 2021.

doi: 10.21037/fomm-2021-02

View this article at: http://dx.doi.org/10.21037/fomm-2021-02

\section{Introduction}

Osteoradionecrosis (ORN) following radiation therapy is a much-feared complication of the treatment of head and neck cancer. As currently defined, ORN is an area of exposed, devitalized irradiated bone that fails to heal over a period of 3-6 months in the absence of local neoplastic disease (1). The incidence varies from $2 \%$ to $37 \%$ depending on the series quoted. Nabil and Samman estimated the risk as being 2-7\% with hyperfractionation and mandible lesions showing elevated risk. Studer $e t$ al. found that in the mandible, marginal or periosteal resection as carrying the highest risk in post-surgical patients. They also identified radiation doses greater than $60 \mathrm{~Gy}$ as the threshold at which ORN developed in the mandible (2). The severity of the condition can range from loss of soft tissues with spontaneous recovery to exposure of necrotized bone for 6 months or more, infection, disabling trismus, persistent pain, skin fistulae and pathological fracture. Early stage ORN can be managed conservatively, aiming to limit progression with medical therapy [antibiotics, pentoxifylline-tocopherol, hyperbaric oxygen (HBO)] and limited surgery. There is general agreement that the most severe cases are best managed surgically with free flap reconstruction replacing the necrotic and poorly vascularized tissues (3).

The aims of resection and free flap reconstruction can 
be described as three-fold: curative, with resection of the diseased tissue; functional, by restoring articulation, mastication and swallowing; and esthetic (3).

Free flap reconstruction in the ORN patient has specific challenges for the surgeon. First of all, it is important, that the possibility of recurrence of neoplasm is ruled out. The surgeon is then faced with a hostile surgical environment with scarring, fibrosis and local infection and or bacterial colonization. Vessels for anastomosis may depleted from previous neck dissection or vascular anastomosis and may exhibit various degrees of radiation damage. Finding appropriate recipient vessels for anastomosis can be challenging and preparing them may be difficult. Friendlander et al. found that radiation doses sufficient to cause ORN can result in atherosclerotic lesions in vessels as large as the carotid. The use of vessels from the contralateral non-irradiated neck has been suggested by some surgeons $(3,4)$.

From 2014 to 2020 the Head and Neck Microvascular unit of the Oral and Maxillofacial Surgery department at Jackson Health/University of Miami treated 149 stage 3 or advanced ORN patients with free flap reconstruction. In this paper we present our approach to reconstruction in these patients and the lessons we have learned.

\section{Preoperative assessment}

Clinical examination is key successful free flap surgery in the ORN patient. The following should be noted and recorded in the assessment.

(I) Presence of fistulas or obvious infection;

(II) Degree of fibrosis and scarring in the neck;

(III) Whether facial hair growth is noted in the planned anastomotic field;

(IV) Presence of trismus, or pathological fracture;

(V) Decrease or loss of sensation of the lower lip or chin.

Significant radiation damage of the bone and the overlying soft tissue (Figures 1,2).

It is extremely important that recurrent malignant disease is ruled out with biopsies if necessary as up to $3.4 \%$ of ORN resection specimens may contain malignancy (3). The patient should be optimised with antibiotics to control infection, as well as oral hygiene.

\section{Imaging}

A good computed tomography (CT) of the facial bones is essential in visualizing the anatomy of the operation field as well as delineating the extent of the ORN. At the University of Miami we use either high resolution cone beam CT or medical grade CT.

It is not our usual practice to routinely order a CT angiogram of the neck vasculature in ORN patients. We have found that this imaging modality often flatters to deceive with "good vessels" on the scan sometimes demonstrating poor calibre and flow or significant atherosclerosis under the microscope. We do however advocate for computed tomography angiography (CTA) in the neck that has been subjected to multiple operations or is clinically "woody" hard on examination.

\section{Perioperative care planning}

Airway management, and patient nutrition should be planned before the operation. It is our preference to determine whether the patient will have a tracheotomy or percutaneous endoscopic gastrostomy (PEG) tube at the office planning visit. Dysphagia and trismus are common complications in radiotherapy of the head and neck, and the degree to which these will be issues should be determined and planned for prior to the free flap surgery. Where possible, we prefer to have PEG tube placement performed prior to the free flap with the tracheotomy performed on the day of surgery.

Where patients have scheduled medical treatment for their ORN (pentoxifylline-tocopherol, antibiotics) these should continue until the day of surgery. The decision on whether to continue treatment post operatively depends on whether we suspect that there are other at-risk areas that are out of the planned resection field.

\section{Virtual surgical planning}

The authors use virtual surgical planification where practical. At our institution we have found that virtual surgical planning significantly decreases operating time as well as aids in planning the resection and inset (Figure 3).

\section{Surgery in the irradiated neck}

We prefer to use a two-team approach as this decreases the total operating time, and mitigates surgeon exhaustion.

Identification of the vessels for anastomosis is done as early as possible. They are prepared minimally, due to their susceptibility to injury. It is best to identify primary 

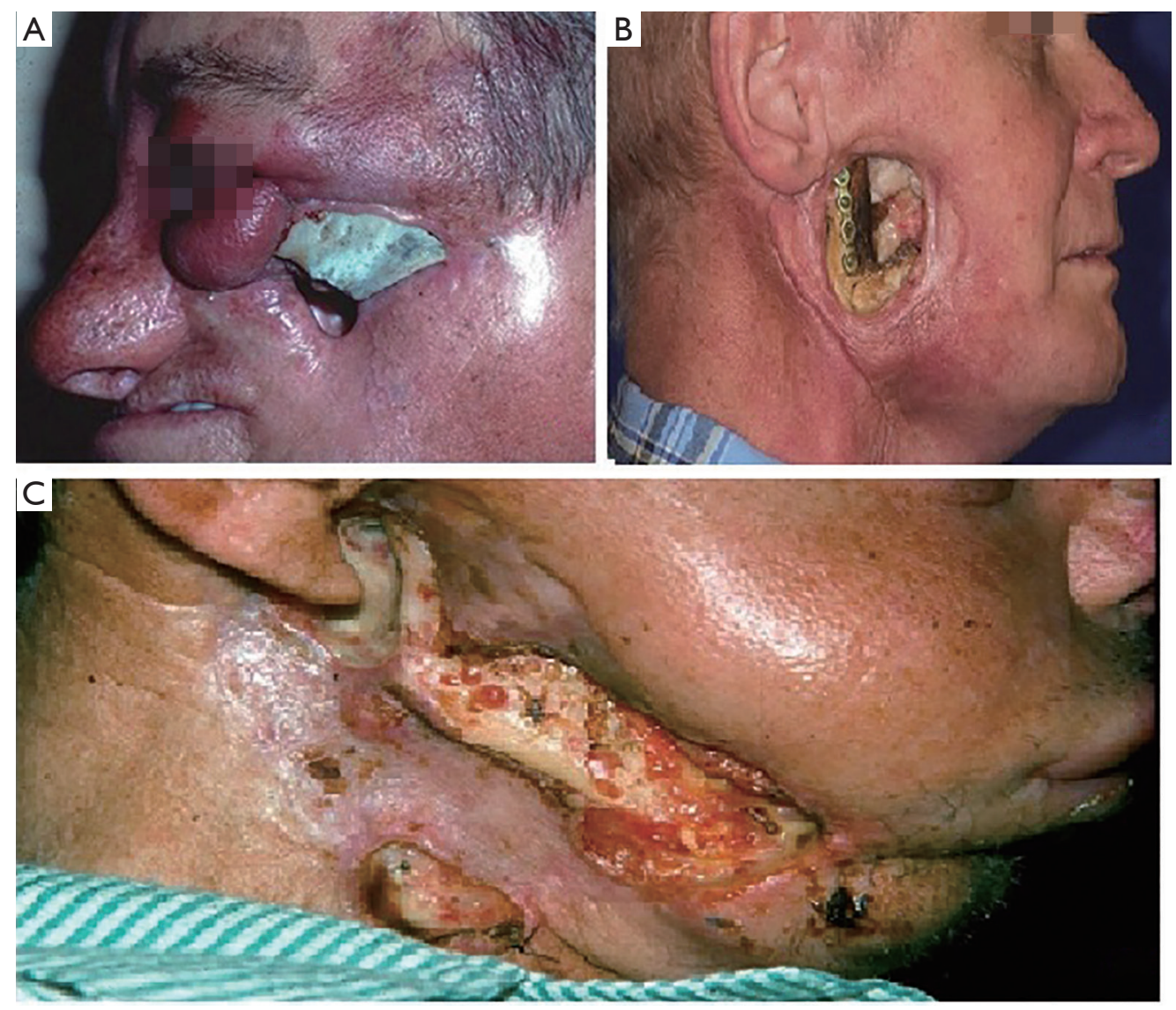

Figure 1 ORN with extensive bone exposure and soft tissue. (A) Zygoma; (B) mandible; (C) neo-mandible (fibula free flap). ORN, osteoradionecrosis.

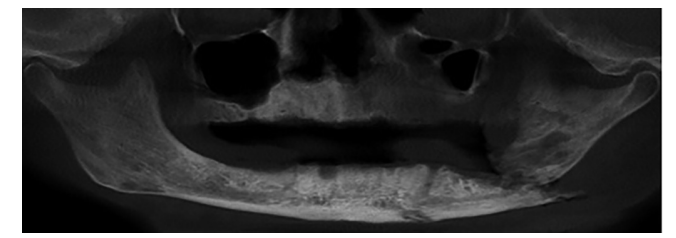

Figure 2 ORN of the left mandible with pathological fracture and loss of sensation in the distribution of the inferior alveolar nerve. ORN, osteoradionecrosis.

as well as secondary options for vascular anastomosis. Where possible utilise the ipsilateral neck vessels. Consider using vessels in the contralateral neck in the following circumstances:

(I) Infection and fistula in the ipsilateral neck;

(II) Excessive fibrosis and a "woody hard" ipsilateral neck.
It is preferable to complete vessel preparation and ablation of the diseased tissue prior to division of the flap pedicle at the flap harvest site. The flap pedicle is divided in manner so as to create as much slack as possible in the neck, avoiding tension in the vessels on anastomosis and more importantly when the patient subjects the neck to the normal range of motion (Figures 4,5).

\section{Vessel anastomosis}

Avoiding vessel trauma is the key to success. We avoid using double micro-clamps to minimize vessel trauma. The vessels should be handled with care avoiding stretching and excessive dilatation with micro instruments (this minimizes intimal tearing).

We utilize the "posterior wall first-continuous interrupted-airborne (PCA) technique" combining three different techniques for vessel anastomosis. We have 

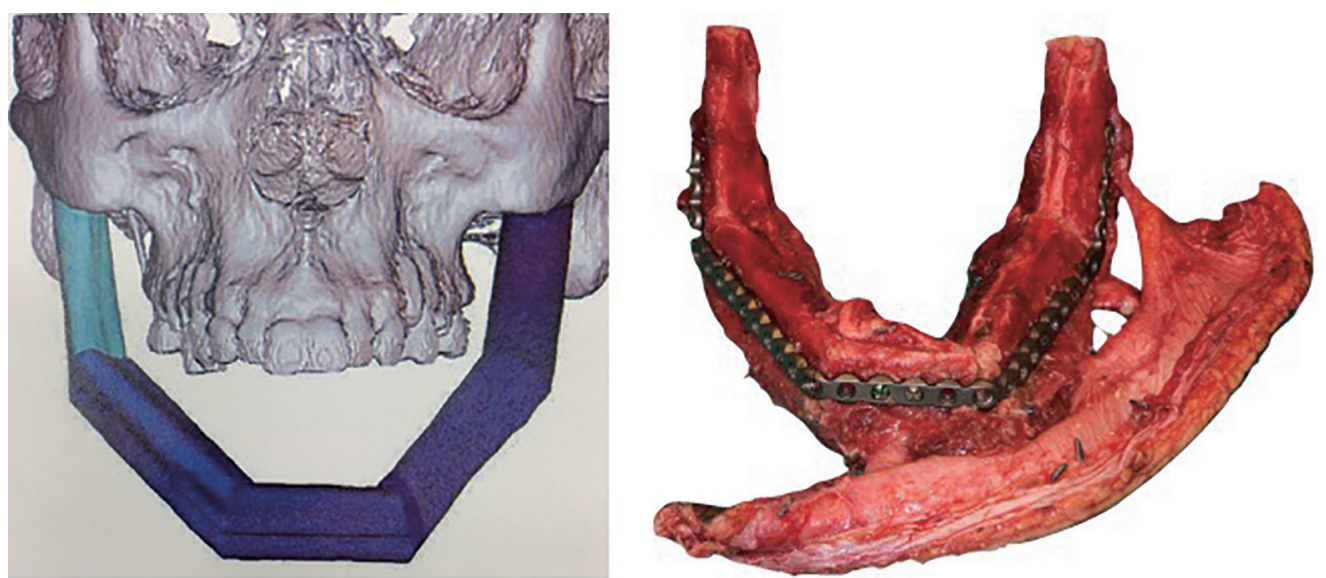

Figure 3 Example of virtual surgical planning for reconstruction of ORN with fibula free flap. ORN, osteoradionecrosis.
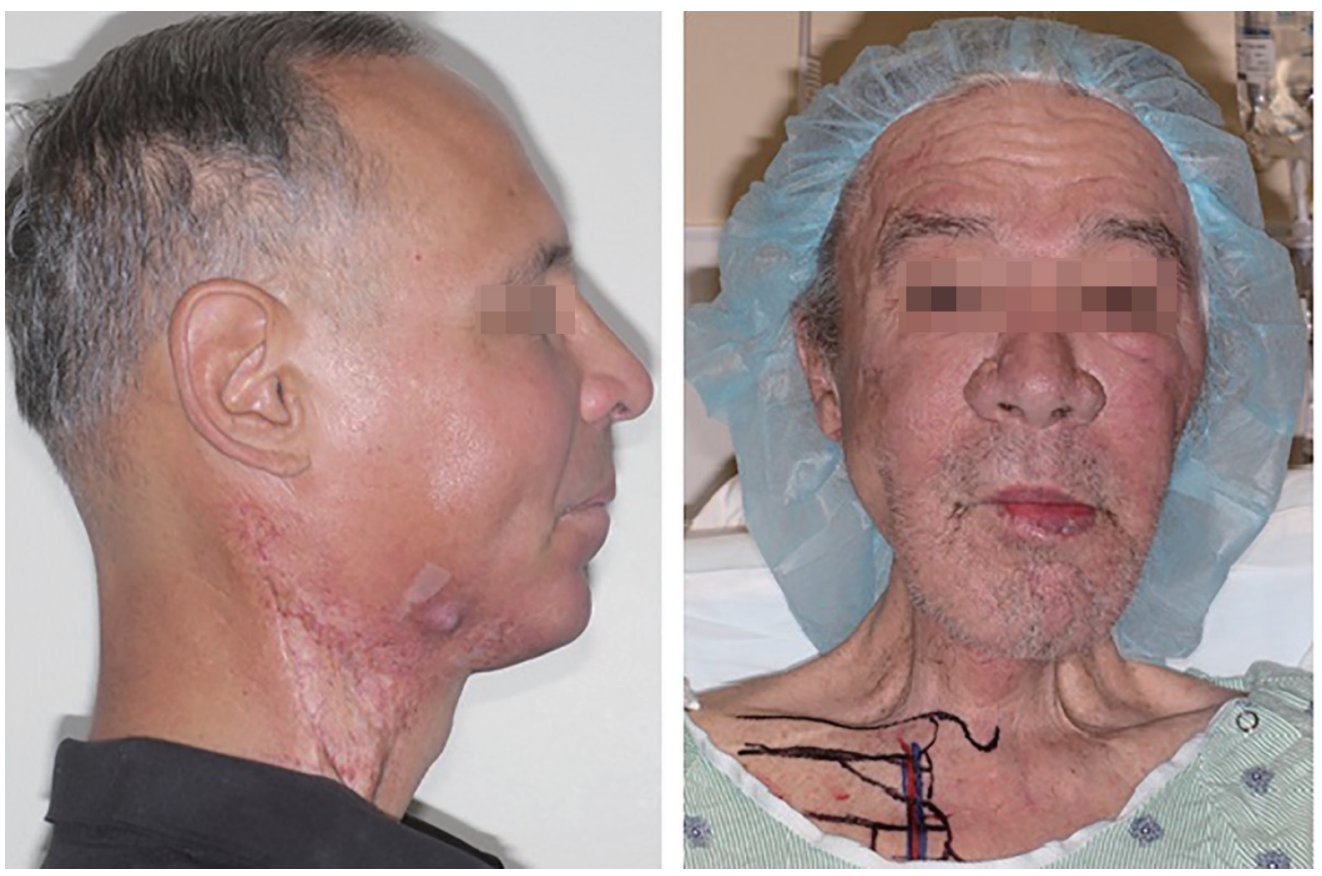

Figure 4 Managment of neck depletion vessels. (A) Severe fibrosis and fistulas of the right neck; (B) vessel depleted neck with plan for internal mammary anastomosis.

found that performing "posterior wall first", continuous interrupted and airborne, is less traumatic to the vessels and also decreases the anastomosis time significantly. Performing the posterior wall anastomosis first eliminates the need to turn the micro-clamps over. The continuous interrupted suture allows for visibility of the lumen throughout the anastomosis preventing "back walling" of the suture as well as trauma to the vessel. Finally, the "airborne" tying technique decreases the suture tying time. The anastomosis is performed in an "in" to "out" direction to minimize the risk of intimal separation.

\section{Anti-coagulation}

Where a patient has been on anti-platelet medication (aspirin or clopidogrel), continue on these medications post 

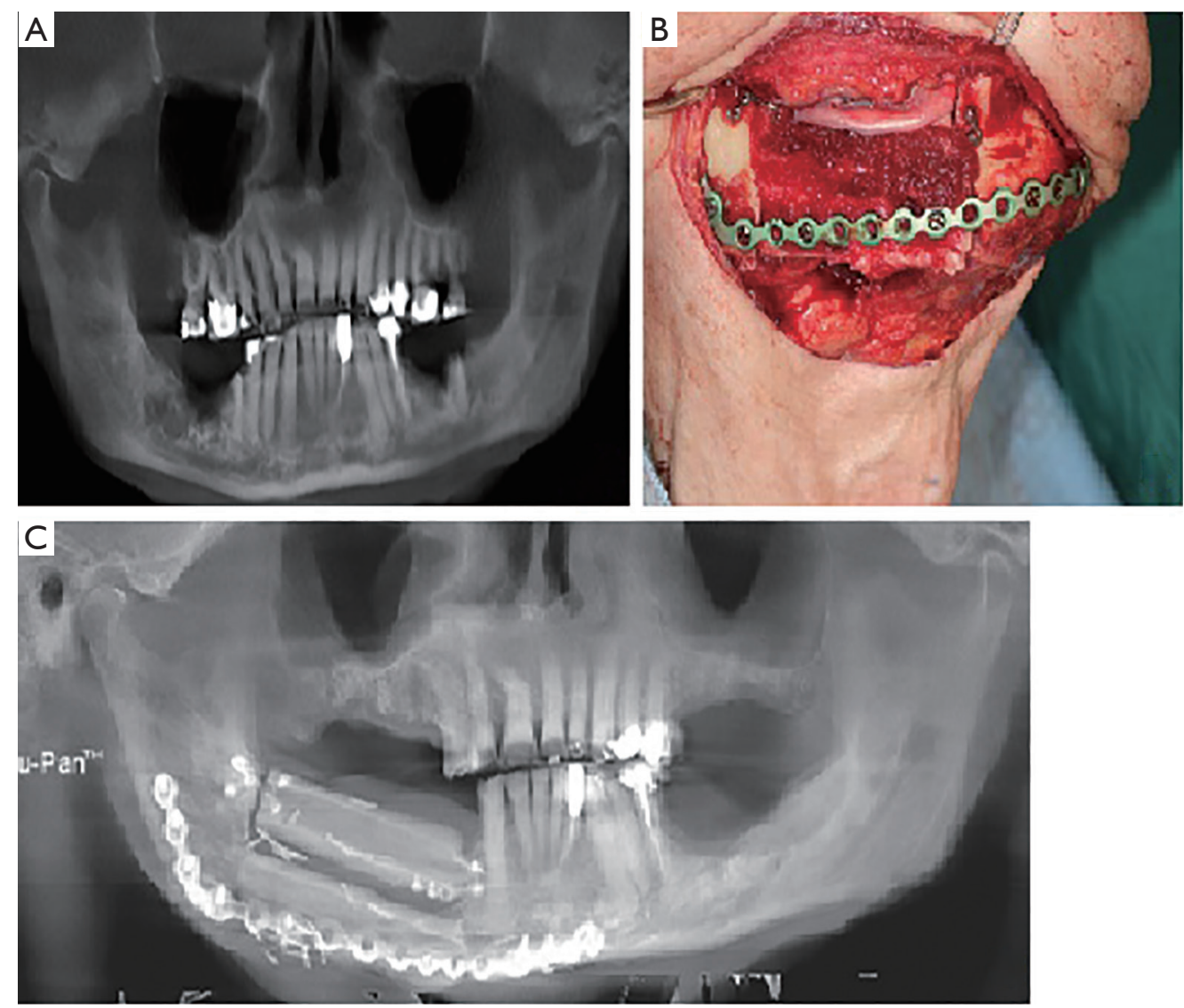

Figure 5 Managment of severe ORN. (A) Stage III ORN of the right mandible; (B) segmental resection of the necrotic bone and reconstruction with a fibula free flap in addition to reconstruction of the inferior alveolar nerve; (C) post-operative panoramic X-ray. ORN, osteoradionecrosis.

operatively. In cases of severe atherosclerosis we administer 3,000 units of intravenous (IV) heparin 5 minutes prior to harvest.

\section{Results}

From 2014 to 2020 the Head and Neck Microvascular unit of the Oral and Maxillofacial Surgery Service at Jackson Health/University of Miami treated 149 ORN patients with free flap reconstruction.

The fibula free flap $(n=65)$ and anterior lateral thigh free flap $(n=61)$ were the workhorse flaps in our series comprising $44 \%$ and $41 \%$ of the total flap surgeries. A total of 13 cases $(8.7 \%)$ involved performing 2 flaps in the same operation. Thirty cases had recorded complications (20.1\%) with one flap loss $(0.7 \%)$ and one case of partial necrosis
$(0.7 \%)$ of the flap. The most common complications encountered were flap congestion with 8 cases $(5.4 \%)$, 5 cases of flap dehiscence, 4 cases of neck hematoma (2.7\%) and 3 cases of infection of the flap reconstruction site (2.0\%). The average length of stay was 8.2 days (Tables 1,2$)$.

\section{Discussion}

Free flap surgery in the ORN patient has significant challenges. Previously performed neck dissection by itself has a strong correlation with free flap loss, wound infection and microvascular revision (5). Previous irradiation of the neck is independently associated with infection and free flap loss. The ORN free flap patient will usually have had a neck dissection and may indeed have wound infection.

Benatar et al. reviewed the impact of pre-operative 
Table 1 Free flap type and number

\begin{tabular}{lc}
\hline Free flap & Number \\
\hline Fibula & 65 \\
Anterior lateral thigh & 61 \\
Tensor fascia lata & 7 \\
Radial & 6 \\
Scapula & 2 \\
Anterior medial thigh & 2 \\
Vastus lateralis & 1 \\
DCIA & 1 \\
Deep inferior epigastric & 1 \\
\hline
\end{tabular}

DCIA, deep circumflex iliac artery.

Table 2 Flap complications by type

\begin{tabular}{lcc}
\hline Complication & Number & Percentage \\
\hline Flap congestion & 8 & $5.4 \%$ \\
Flap dehiscence & 5 & $3.4 \%$ \\
Neck hematoma & 4 & $2.7 \%$ \\
Infection of reconstruction site & 3 & $2.0 \%$ \\
ORN recurrence & 2 & $1.3 \%$ \\
Abandoned flap surgery & 1 & $0.7 \%$ \\
Complete flap loss & 1 & $0.7 \%$ \\
Partial flap necrosis & 1 & $0.7 \%$ \\
Stroke & 1 & $0.7 \%$ \\
Hypoglossal nerve injury & 1 & $0.7 \%$ \\
Airway obstruction (mucus plug) & 1 & $0.7 \%$ \\
Unspecified & 1 & $0.7 \%$ \\
\hline
\end{tabular}

ORN, osteoradionecrosis.

radiation on free flap reconstruction in the head and neck (5). A total of 136 cases with previous radiotherapy were included in their series. The rate of flap failure recorded was $14 \%$ with patient death at $3 \%$. Their series noted a local complication rate of $40 \%$ in the head and neck free flap with the most common complications noted being fistula (23\%), hematoma (21\%)and infection (7\%). Average length of stay in their series was 27 days. These outcomes were considerably worse where patients with more than 60 Gy of radiation treatment had been given. In this cohort flap failure was $21 \%$ with death at $4 \%$. Local complication rates were $57 \%$ with the most common being fistula formation (43\%), infection (36\%) and hematoma (21\%). Length of stay in this cohort was 41 days.

Zaghi et al. recorded 73 cases of free flap reconstruction over their 17-year series (6). They recorded no free flap failures and a partial necrosis rate of $2.7 \%$. Major complications (resulting in increased length of stay greater than 20 days) were 8 cases (11.0\%). Minor complications were 21 cases (28.8\%). Average length of stay in their series was 12 days.

Patients in our series performed considerably well in comparison to the outcomes recorded in the literature. All but four of the 149 patients received total doses over $50 \mathrm{~Gy}$. The free flap success rate was over $98 \%$. Free flap surgery was abandoned in one case where no suitable vessels were identified in the neck for anastomosis. The single case of flap loss was in a patient with a vessel deplete neck, and multiple medical comorbidities. The same patient also sustained a stroke. This case is a particularly good illustration of the potential risks involved in surgery in these complex patients.

\section{Conclusions}

ORN is an increasing indication for free flap surgery. Though this surgery is complex and has greater risks than free flap surgery in the "virgin" neck, our experience demonstrates that good outcomes can be achieved consistently with a structured approach.

\section{Acknowledgments}

Funding: None.

\section{Footnote}

Provenance and Peer Review: This article was commissioned by the Editorial Office, Frontiers of Oral and Maxillofacial Medicine for the series "Microvascular reconstruction of head and neck oncological defects-state of the art". The article has undergone the external peer review.

Conflicts of Interest: All authors have completed the ICMJE uniform disclosure form (available at https://fomm. amegroups.com/article/view/10.21037/fomm-2021-02/coif). The series "Microvascular reconstruction of head and neck oncological defects-state of the art" was commissioned by the editorial office without any funding or sponsorship. PC served as the unpaid Guest Editor of the series and serves 
as an unpaid editorial board member of Frontiers of Oral and Maxillofacial Medicine from Apr 2020 to Mar 2022. RT and AK are compensated consultants for Axogen Inc. The authors have no other conflicts of interest to declare.

Ethical Statement: The authors are accountable for all aspects of the work in ensuring that questions related to the accuracy or integrity of any part of the work are appropriately investigated and resolved.

Open Access Statement: This is an Open Access article distributed in accordance with the Creative Commons Attribution-NonCommercial-NoDerivs 4.0 International License (CC BY-NC-ND 4.0), which permits the noncommercial replication and distribution of the article with the strict proviso that no changes or edits are made and the original work is properly cited (including links to both the formal publication through the relevant DOI and the license). See: https://creativecommons.org/licenses/by-nc-nd/4.0/.

\section{References}

1. Nabil S, Samman N. Risk factors for osteoradionecrosis

doi: 10.21037/fomm-2021-02

Cite this article as: Kaleem A, Amailuk P, Cariati P, Tursun R. Free flap surgery in osteoradionecrosis of the head and neck. Front Oral Maxillofac Med 2021;3:13. after head and neck radiation: a systematic review. Oral Surg Oral Med Oral Pathol Oral Radiol 2012;113:54-69.

2. Studer G, Studer SP, Zwahlen RA, et al. Osteoradionecrosis of the mandible: minimized risk profile following intensity-modulated radiation therapy (IMRT). Strahlenther Onkol 2006;182:283-8.

3. Chrcanovic BR, Reher P, Sousa AA, et al. Osteoradionecrosis of the jaws--a current overview-Part 2: dental management and therapeutic options for treatment. Oral Maxillofac Surg 2010;14:81-95.

4. Wong JK, Wood RE, McLean M. Conservative management of osteoradionecrosis. Oral Surg Oral Med Oral Pathol Oral Radiol Endod 1997;84:16-21.

5. Benatar MJ, Dassonville O, Chamorey E, et al. Impact of preoperative radiotherapy on head and neck free flap reconstruction: a report on 429 cases. J Plast Reconstr Aesthet Surg 2013;66:478-82.

6. Zaghi S, Danesh J, Hendizadeh L, et al. Changing indications for maxillomandibular reconstruction with osseous free flaps: a 17-year experience with 620 consecutive cases at UCLA and the impact of osteoradionecrosis. Laryngoscope 2014;124:1329-35. 